TRANSACTIONS OF THE

AMERICAN MATHEMATICAL SOCIETY

Volume 354, Number 5, Pages 2035-2053

S 0002-9947(02)02915-X

Article electronically published on January 8, 2002

\title{
A SEMIGROUP OF OPERATORS IN CONVEXITY THEORY
}

\author{
CHRISTER O. KISELMAN
}

\begin{abstract}
We consider three operators which appear naturally in convexity theory and determine completely the structure of the semigroup generated by them.

Resumo. Duongrupo de operatoroj en la teorio pri konvekseco. Ni konsideras tri operatorojn kiuj aperas nature en la teorio pri konvekseco kaj plene determinas la strukturon de la duongrupo generita de ili.
\end{abstract}

\section{INTRODUCTION}

The simplest convex functions on a vector space are the affine functions, and it is of interest to represent a convex function as a supremum of these simple functions. Such a representation is possible only if the function has three properties: it must be convex; it must be lower semicontinuous with respect to an appropriate topology; and it cannot take the value minus infinity unless it is identically minus infinity. Conversely, using the Fenchel transformation one can prove that these three properties are also sufficient for such a representation to hold; indeed, the function is then equal to its second Fenchel transform, or second conjugate function.

There are thus three operations naturally associated with the Fenchel transformation: that of taking the largest convex minorant; that of taking the largest lower semicontinuous minorant; and that of taking the constant minus infinity if the function attains that value and leaving it unchanged otherwise. These three operations generate a semigroup in the semigroup of all operators on the set of functions on the vector space in question. How many elements are there in this semigroup? What structure does it have with respect to composition and with respect to the natural order? Is it a lattice under this order? Is there a representation of the semigroup as a semigroup of matrices? The purpose of this note is to provide answers to these questions. A quick look at Figure 1 will reveal the order structure of the semigroup.

We now define the three operations more precisely. Let $E$ be a real vector space. To any function $f$ on $E$ with values in the extended real line $\mathbf{R} \cup\{-\infty,+\infty\}=$ $[-\infty,+\infty]$ we associate its convex hull or largest convex minorant $c(f)$, defined as the supremum of all its convex minorants. It can be shown that

$$
c(f)(x)=\inf \left(\sum_{1}^{N} \lambda_{j} f\left(x_{j}\right) ; N \geqslant 1, \sum_{1}^{N} \lambda_{j} x_{j}=x\right), \quad x \in E,
$$

Received by the editors March 21, 2001 and, in revised form, July 8, 2001.

2000 Mathematics Subject Classification. Primary 20M20, 20M30, 26B25, 52A41, 47H20.

(C)2002 American Mathematical Society 
where the infimum is taken over all representations of $x$ as a barycenter of finitely many points $x_{j}$ with $f\left(x_{j}\right)<+\infty, j=1, \ldots, N, N \geqslant 1$, and taking positive weights $\lambda_{j}$ with sum equal to 1 .

The second operator is that of taking the largest lower semicontinuous minorant of the function, thus the supremum of all its lower semicontinuous minorants for some topology $\tau$ on $E$, also given by the formula

$$
l(f)(x)=\liminf _{y \rightarrow x} f(y), \quad x \in E .
$$

It is not difficult to see that this operation corresponds to taking the closure of the epigraph of the function, i.e., epi $(l(f))=\overline{\text { epi } f}$, where the closure is taken with respect to the Cartesian product of $\tau$ and the usual topology on $\mathbf{R}$.

In Fenchel duality we consider a vector space $F$ of linear forms on $E$, thus a subspace of the algebraic dual $E^{*}$ of $E$. The topologies that are of interest are $\sigma(E, F)$, the weakest topology on $E$ such that all linear forms in $F$ are continuous, and $\sigma(F, E)$, the weakest topology on $F$ such that all evaluation mappings $F \ni$ $\xi \mapsto \xi(x), x \in E$, are continuous. We can for instance choose $F=E^{*}$, or $F=E^{\prime}$, the topological dual of $E$ under a given topology. We do not require that $E$ and $F$ be in duality; it is even allowed to take $F=\{0\}$.

The third operator is the operator $m$, defined as

$$
m(f)(x)= \begin{cases}f(x), & \text { if } f \text { is everywhere }>-\infty \\ -\infty, & \text { if } f \text { assumes the value }-\infty\end{cases}
$$

The three operators $c, l, m$ generate a semigroup $G(E)$ with composition as multiplication.

The plan of the paper is as follows. First we study operations on functions defined on vector spaces (Section 2). Next we define an abstract semigroup $\mathcal{G}$ of eighteen elements generated by the identity and three elements $c, l, m$ subject to the relations $c^{2}=c, l^{2}=l, m^{2}=m$, and $c l c=l c l=l c, c m c=m c m=m c$, $l m l=m l m=m l$ (Theorem 3.2). We find a matrix representation of this semigroup using $3 \times 3$ matrices (Theorem 3.3 ). For every vector space $E$, the semigroup $G(E)$ is then a homomorphic image of $\mathcal{G}$, and we determine this homomorphic image for spaces of dimension zero, one, two and higher (Theorem 4.1).

The order of $G(E)$ can be $1,6,15,16,17$, or 18 , depending on the dimension of $E$ and its topology. The order is 15 for $E=\mathbf{R}, 16$ for $E=\mathbf{R}^{n}, n \geqslant 2$, and 18 for every normed space $E$ of infinite dimension.

I am grateful to Sten Kaijser for helpful comments concerning matrix representation of semigroups, in particular for bringing the paper by Hewitt and Zuckerman 1955] to my attention. Svante Janson carefully read an early draft of the paper and saved me from some embarrassing errors; I am very grateful to him.

\section{Closure operators and Galois Correspondences}

An order relation in a set $X$ is a relation (a subset of $X \times X$ ) which satisfies three conditions: it is reflexive, antisymmetric and transitive. This means, if we denote the relation by $\leqslant$, that for all $x, y, z \in X$,

$$
\begin{gathered}
x \leqslant x ; \\
x \leqslant y \text { and } y \leqslant x \text { imply } x=y ; \\
x \leqslant y \text { and } y \leqslant z \text { imply } x \leqslant z .
\end{gathered}
$$


An ordered set is a set $X$ together with an order relation. (Sometimes one says partially ordered set.)

A basic example is the set $P(W)$ of all subsets of a set $W$, with the order relation given by inclusion, thus $A \leqslant B$ being defined as $A \subset B$ for $A, B \in P(W)$.

A closure operator in an ordered set $X$ is a mapping $X \ni x \mapsto \bar{x} \in X$ which is expanding (or extensive), increasing (or isotone, order preserving), and idempotent; in other words, which satisfies the following three conditions for all $x, y \in X$ :

$$
\begin{gathered}
x \leqslant \bar{x} ; \\
x \leqslant y \text { implies } \bar{x} \leqslant \bar{y} ; \\
\overline{\bar{x}}=\bar{x} .
\end{gathered}
$$

The element $\bar{x}$ is said to be the closure of $x$. Elements $x$ such that $\bar{x}=x$ are called closed (for this operator). An element is closed if and only if it is the closure of some element (and then it is the closure of itself).

A basic example of a closure operator is of course the topological closure operator which associates to a set in a topological space its topological closure, i.e., the smallest closed set containing the given set.

Another closure operator of great importance is the operator which associates to a set in $\mathbf{R}^{n}$ its convex hull, the smallest convex set containing the given set.

A Galois correspondence is a pair $(f, g)$ of two decreasing mappings $f: X \rightarrow Y$, $g: Y \rightarrow X$ of two given ordered sets $X, Y$ such that $g \circ f$ and $f \circ g$ are expanding. In other words we have $f(x) \geqslant f\left(x^{\prime}\right)$ and $g(y) \geqslant g\left(y^{\prime}\right)$ if $x \leqslant x^{\prime}$ and $y \leqslant y^{\prime}$, and $g(f(x)) \geqslant x$ and $f(g(y)) \geqslant y$ for all $x \in X, y \in Y$.

The notion of Galois correspondence originates in Galois theory, which deals with the correspondence between all intermediate subfields of a field extension and the set of subgroups of the Galois group of the extension. The notion was introduced in the general setting of ordered sets by Oystein Ore [1944, 495] under the name of Galois correspondence or Galois connexion. (Sometimes he calls each of the mappings $f$ and $g$ a correspondence, the two of them together a connexion.) See also Everett [1944, 516], Birkhoff [1948, 56, 57], and Kuroš [1962, 6:11].

Proposition 2.1 (Ore 1944, 496]). Let $f: X \rightarrow Y, g: Y \rightarrow X$ be a Galois correspondence. Then $g \circ f: X \rightarrow X$ and $f \circ g: Y \rightarrow Y$ are closure operators. Moreover, $f \circ g \circ f=f$ and $g \circ f \circ g=g$.

Proof. That $g \circ f$ and $f \circ g$ are expanding is part of the definition of a Galois correspondence; that they are increasing follows from the fact that they are compositions of two decreasing mappings. We know that $f \circ g$ is expanding, so $(f \circ g)(f(x)) \geqslant f(x)$; thus $f \circ g \circ f \geqslant f$. On the other hand, also $g \circ f$ is expanding, i.e., $g \circ f \geqslant \operatorname{id}_{X}$, so $f \circ g \circ f \leqslant f \circ \operatorname{id}_{X}=f$, hence $f \circ g \circ f=f$. By symmetry, $g \circ f \circ g=g$. From either one of these identities we easily obtain that $g \circ f$ and $f \circ g$ are idempotent.

It is now natural to ask whether the closure operators one obtains from Galois correspondences have some special property. The answer is no: every closure operator comes in a trivial way from some Galois correspondence.

Proposition 2.2. Let $x \mapsto \bar{x}$ be a closure operator defined in an ordered set $X$. Then there exist an ordered set $Y$ and a Galois correspondence $f: X \rightarrow Y, g: Y \rightarrow$ $X$ such that $\bar{x}=g(f(x))$ for all $x \in X$. 
Proof. We define $Y$ as the set of all closed elements in $X$ with the opposite order; thus $y \leqslant_{Y} y^{\prime}$ shall mean that $y \geqslant_{X} y^{\prime}$. Let $f: X \rightarrow Y$ and $g: Y \rightarrow X$ be defined by $f(x)=\bar{x}$ and $g(y)=y$. Then both $f$ and $g$ are decreasing, and $g \circ f(x)=\bar{x} \geqslant_{X} x$, $f \circ g(y)=y \geqslant_{Y} y$. So $g \circ f$ and $f \circ g$ are expanding, and $\bar{x}=g(f(x))$ as desired.

Proposition 2.2 is, in a sense, completely uninteresting. This is because the Galois correspondence is obtained from $X$ and the closure operator in a totally trivial way. However, there are many Galois correspondences in mathematics that are highly interesting and represent a given closure operator. This is so because they allow for important calculations to be made or for new insights into the theory.

We now ask whether the composition of two closure operators is a closure operator. It is for instance well-known that if we first take the topological closure of a set and then its convex hull, we get an operator which is not idempotent, thus not a closure operator.

Proposition 2.3. Let $f, g: X \rightarrow X$ be two closure operators. The following properties are equivalent:

(a) $g \circ f$ is a closure operator;

(b) $g \circ f$ is idempotent;

(c) $g \circ f \circ g=g \circ f$;

(d) $f \circ g \circ f=g \circ f$;

(e) $g(x)$ is $f$-closed if $x$ is $f$-closed.

If one of these conditions is satisfied, then $g \circ f$ is the supremum of the two closure operators $f$ and $g$ in the ordered set of all closure operators; moreover $f \circ g \leqslant g \circ f$.

Proof. That $h=g \circ f$ is expanding and increasing is true for any composition of expanding and increasing mappings, so it is clear that (a) and (b) are equivalent. It is also easy to see that (d) and (e) are equivalent. If (c) holds, then $h \circ h=$ $g \circ f \circ g \circ f=g \circ f \circ f=g \circ f=h$, so $h$ is idempotent. Similarly, (d) implies (b). Conversely, if (b) holds, then

$$
g \circ f \leqslant g \circ f \circ g \leqslant h \circ h=h=g \circ f
$$

and

$$
g \circ f \leqslant f \circ g \circ f \leqslant h \circ h=h=g \circ f,
$$

so we must have equality all the way in both chains of inequalities, which proves that (c) and (d) hold. The last statement is easy to verify.

Corollary 2.4. Two closure operators $f$ and $g$ commute if and only if both $g \circ f$ and $f \circ g$ are closure operators.

Proof. If $g \circ f=f \circ g$, then (c) obviously holds, so $g \circ f$ is a closure operator. Conversely, if $g \circ f$ is a closure operator, then (c) applied to $f$ and $g$ says that $g \circ f \circ g=g \circ f$; if also $f \circ g$ is a closure operator, then (d) applied to $g$ and $f$ says that $g \circ f \circ g=f \circ g$. Thus $f$ and $g$ commute.

When two closure operators $f$ and $g$ are given, it may happen that $f \leqslant g$. Then the semigroup generated by $f$ and $g$ consists of at most three elements: id $x, f, g$. If both $g \circ f$ and $f \circ g$ are closure operators, then the semigroup generated by $f$ and $g$ has at most four elements: $\operatorname{id}_{X}, f, g$, and $g \circ f=f \circ g$. If precisely one of $g \circ f$ and $f \circ g$ is a closure operator, then the semigroup generated has exactly five elements, $\operatorname{id}_{X}, f, g, g \circ f$, and $f \circ g$, of which four are closure operators. When neither $g \circ f$ 
nor $f \circ g$ is a closure operator, the semigroup of all compositions $f_{m} \circ \cdots \circ f_{1}$, with $f_{j}=f$ or $f_{j}=g, m \in \mathbf{N}$, may be finite or infinite.

Applying Proposition 2.3 to the case of the two operators $f(A)=\operatorname{cvx} A$ and $g(A)=\bar{A}$, we see that the operation of taking the topological closure of the convex hull, $A \mapsto \overline{\operatorname{cvx} A}$, is a closure operator. Indeed, it can be proved that the closure (with respect to a reasonable topology; see Proposition 2.5 below) of a convex set is always convex, so condition (e) is satisfied. (On the other hand, the convex hull of a closed set is not always closed.) We call $\overline{\operatorname{cvx} A}$ the closed convex hull of $A$. This is a case where the semigroup generated by $f$ and $g$ consists of five elements.

Next we consider three closure operators $f, g, h$ such that one of $f \circ g$ and $g \circ f$ is a closure operator, and similarly for the other pairs $\{g, h\},\{h, f\}$. Up to renaming the operators, there are then only two cases. Either $g \circ f, h \circ g$ and $h \circ f$ are closure operators (alphabetical order), or $g \circ f, h \circ g$ and $f \circ h$ are closure operators (cyclic order). It turns out that the operators defined by (1.1)-(1.3) belong to the first case. More precisely we have:

Proposition 2.5. Let $c, l, m$ denote the three operators defined by (1.1)-(1.3) on a vector space $E$ equipped with a topology $\tau$. They are all closure operators, in particular they are idempotent:

$$
c \circ c=c, \quad l \circ l=l, \quad m \circ m=m .
$$

Moreover, they satisfy

$$
c \circ m \circ c=m \circ c \circ m=m \circ c, \quad l \circ m \circ l=m \circ l \circ m=m \circ l,
$$

which means that the $m$-closure of a convex function is convex and that the $m$ closure of a lower semicontinuous function is lower semicontinuous. If the topology $\tau$ is such that all translations $x \mapsto x-a, a \in E$, and all dilations $x \mapsto \lambda x, \lambda \in \mathbf{R}$, are continuous, then we also have

$$
c \circ l \circ c=l \circ c \circ l=l \circ c
$$

which means that the largest lower semicontinuous minorant of a convex function is convex.

Proof. All statements except perhaps the last have routine proofs. Let $f$ be a convex function on $E$ and define $g=l(f)$. To prove that $g$ is convex, we fix $x_{j} \in E$ such that $g\left(x_{j}\right)<+\infty, j=0,1$, and a number $\left.t \in\right] 0,1[$, and shall then prove that $g\left((1-t) x_{0}+t x_{1}\right) \leqslant(1-t) g\left(x_{0}\right)+t g\left(x_{1}\right)$. Given any numbers $A_{j}>g\left(x_{j}\right)$ and any neighborhood $U$ of $x=(1-t) x_{0}+t x_{1}$, we can find a neighborhood $U_{0}$ of $x_{0}$ such that $(1-t) U_{0}+t x_{1} \subset U$. By the definition of $g=l(f)$, there exists a point $y_{0} \in U_{0}$ such that $f\left(y_{0}\right)<A_{0}$. Next we can find a neighborhood $U_{1}$ of $x_{1}$ such that $(1-t) y_{0}+t U_{1} \subset U$. By the definition of $g=l(f)$ again, there exists a point $y_{1} \in U_{1}$ such that $f\left(y_{1}\right)<A_{1}$. Since $f$ is assumed to be convex, we know that $f\left((1-t) y_{0}+t y_{1}\right) \leqslant(1-t) f\left(y_{0}\right)+t f\left(y_{1}\right)<(1-t) A_{0}+t A_{1}$. Hence $\inf _{y \in U} f(y)<(1-t) A_{0}+t A_{1}$. Since this is true for all choices of the $A_{j}$, we see that $\inf _{y \in U} f(y) \leqslant(1-t) g\left(x_{0}\right)+t g\left(x_{1}\right)$. Finally, since this is true for any neighborhood $U$ of $x$, we get

$$
g(x)=l(f)(x)=\liminf _{y \rightarrow x} f(y)=\sup _{U} \inf _{y \in U} f(y) \leqslant(1-t) g\left(x_{0}\right)+t g\left(x_{1}\right) .
$$

This shows that $l(f)$ is convex. 
It follows from (2.8) and (2.9) that $m \circ c, m \circ l$, and $l \circ c$ are closure operators; cf. Proposition 2.3. We shall see later that in general $c, l$ and $m$ do not commute (Theorem 4.1).

We form all possible compositions of these operators, $f_{k} \circ \cdots \circ f_{2} \circ f_{1}$, where $k \geqslant 0$ and the $f_{j}=c, l$, or $m$. Thus we get a semigroup $G(E)$ in the set of all operators on $[-\infty,+\infty]^{E}$. We shall now investigate its structure. We shall do so by defining first an abstract semigroup $\mathcal{G}$, of which each $G(E)$ is a homomorphic image.

\section{An ABStract SEMigroup OF ORDER EIGHTEEN}

Definition 3.1. We let $\mathcal{G}$ denote the semigroup generated by the identity id $=\mathbf{1}$ and three other elements $c, l, m$ which are subject to the relations

$$
\begin{gathered}
c^{2}=c, \quad l^{2}=l, \quad m^{2}=m ; \\
c l c=l c l=l c, \quad c m c=m c m=m c, \quad l m l=m l m=m l .
\end{gathered}
$$

Theorem 3.2. The semigroup $\mathcal{G}$ defined by Definition 3.1 has eighteen elements, which can be represented by products of the generators $c, l$ and $m$ as follows:

(0) One element using zero factors: id $=\mathbf{1}$;

(i) Three elements using one factor: $c, l, m$;

(ii) Six element using two factors: $c l, c m, l c, l m, m c, m l$;

(iii) Six elements using three factors: $\mathrm{clm}, \mathrm{cml}, \mathrm{lcm}, \mathrm{lmc}, \mathrm{mcl}, \mathrm{mlc}=\mathbf{0}$;

(iv) Two elements using four factors: $l \mathrm{cml}, \mathrm{lmcl}$.

Of these, eight are idempotent, viz. id $=\mathbf{1}, c, l, m, l c, m c, m l, m l c=\mathbf{0}$, while the other ten, viz. $\mathrm{cl}, \mathrm{cm}, \mathrm{lm}, \mathrm{clm}, \mathrm{lcm}, \mathrm{cml}, l \mathrm{cml}, \mathrm{lmc}, \mathrm{mcl}, \mathrm{lmcl}$, are not.

Proof. After the elements representable by zero, one or two factors we form all three-letter combinations. Since it does not pay to repeat a letter immediately in view of $(3.1)$, there are only $3 \cdot 2 \cdot 2=12$ combinations that could possibly represent a new element:

$$
\begin{array}{llll}
c l c, & \quad \mathrm{~lm}, & \mathrm{cmc}, & \mathrm{cml}, \\
\mathrm{lcl}, & \mathrm{lcm}, & \mathrm{lmc}, & \mathrm{lml}, \\
\mathrm{mcl}, & \mathrm{mcm}, & \mathrm{mlc}, & \mathrm{mlm} .
\end{array}
$$

Of these twelve combinations, six are equal to elements representable by two factors:

$$
c l c=l c l=l c ; \quad c m c=m c m=m c ; \quad l m l=m l m=m l .
$$

The remaining six are listed under (iii).

So far we have found sixteen elements, not yet known to be distinct. Using (3.1) and (3.2), we can prove by induction on the length of $a$ that $c a c=a c$ and mam $=m a$ for all $a \in \mathcal{G}$, thus that there is no use in repeating $c$ or $m$. If we multiply the six elements listed under (iii) by $l$ from the left, we get $l \mathrm{clm}=l \mathrm{~cm}$, $l \mathrm{cml}, l l \mathrm{~cm}=l \mathrm{~cm}, l l m c=l m c, l m c l, l m l c=m l c$. So possibly $l \mathrm{cml}$ and $l m c l$ represent new elements. Five or more letters will not give anything new.

Thus we see that $\mathcal{G}$ has at most the eighteen elements listed in (0)-(iv). It remains to be proved that these elements are in fact all different. This will follow from the next theorem, where we represent $\mathcal{G}$ as a semigroup of matrices with exactly eighteen elements. 
Any finite semigroup with a neutral element admits a faithful representation as a semigroup of $n \times n$ matrices, where $n$ is the order of the semigroup; see Hewitt and Zuckerman [1955] Theorem 3.21]. Thus 9 admits, by this general theorem, a representation in terms of $18 \times 18$ matrices. However, we shall see that we can find a representation in terms of $3 \times 3$ matrices.

\section{Theorem 3.3. Define}

$$
I=\left(\begin{array}{lll}
1 & 0 & 0 \\
0 & 1 & 0 \\
0 & 0 & 1
\end{array}\right), \quad C=\left(\begin{array}{lll}
1 & 0 & 1 \\
0 & 1 & 1 \\
0 & 0 & 0
\end{array}\right), \quad L=\left(\begin{array}{lll}
1 & 1 & 0 \\
0 & 0 & 0 \\
0 & 0 & 1
\end{array}\right), \quad M=\left(\begin{array}{lll}
0 & 0 & 0 \\
0 & 1 & 0 \\
0 & 0 & 1
\end{array}\right) .
$$

Then $C, L$ and $M$ satisfy the relations corresponding to (3.1) and (3.2). They generate a semigroup $\mathcal{H}$ of matrices with eighteen elements; in particular the products corresponding to (0)-(iv) are all different.

Proof. It is easy to prove the relations corresponding to (3.1) and (3.2); it follows that $\mathcal{H}$ is a homomorphic image of $\mathcal{G}$.

We may calculate all elements of $\mathcal{H}$. In addition to $I, C, L, M$ they are:

$$
\begin{aligned}
& C L=\left(\begin{array}{lll}
1 & 1 & 1 \\
0 & 0 & 1 \\
0 & 0 & 0
\end{array}\right), \quad C M=\left(\begin{array}{lll}
0 & 0 & 1 \\
0 & 1 & 1 \\
0 & 0 & 0
\end{array}\right), \quad L M=\left(\begin{array}{lll}
0 & 1 & 0 \\
0 & 0 & 0 \\
0 & 0 & 1
\end{array}\right), \\
& C L M=\left(\begin{array}{lll}
0 & 1 & 1 \\
0 & 0 & 1 \\
0 & 0 & 0
\end{array}\right) \\
& L C=\left(\begin{array}{lll}
1 & 1 & 2 \\
0 & 0 & 0 \\
0 & 0 & 0
\end{array}\right), \quad M C=\left(\begin{array}{lll}
0 & 0 & 0 \\
0 & 1 & 1 \\
0 & 0 & 0
\end{array}\right), \quad M L=\left(\begin{array}{lll}
0 & 0 & 0 \\
0 & 0 & 0 \\
0 & 0 & 1
\end{array}\right) \\
& L C M=\left(\begin{array}{lll}
0 & 1 & 2 \\
0 & 0 & 0 \\
0 & 0 & 0
\end{array}\right), \quad L C M L=\left(\begin{array}{lll}
0 & 0 & 2 \\
0 & 0 & 0 \\
0 & 0 & 0
\end{array}\right), \quad C M L=\left(\begin{array}{lll}
0 & 0 & 1 \\
0 & 0 & 1 \\
0 & 0 & 0
\end{array}\right) \\
& L M C=\left(\begin{array}{lll}
0 & 1 & 1 \\
0 & 0 & 0 \\
0 & 0 & 0
\end{array}\right), \quad L M C L=\left(\begin{array}{lll}
0 & 0 & 1 \\
0 & 0 & 0 \\
0 & 0 & 0
\end{array}\right), \quad M C L=\left(\begin{array}{lll}
0 & 0 & 0 \\
0 & 0 & 1 \\
0 & 0 & 0
\end{array}\right) \\
& M L C=\left(\begin{array}{ccc}
0 & 0 & 0 \\
0 & 0 & 0 \\
0 & 0 & 0
\end{array}\right)
\end{aligned}
$$

Since they are all different, it follows that $\mathcal{H}$ has eighteen elements. Therefore $\mathcal{H}$ is isomorphic to $\mathcal{G}$.

One forms the abelization of a group by taking the quotient over the subgroup generated by all commutators $g^{-1} h^{-1} g h$. For a semigroup this procedure is not available. Instead we may define the abelization of a semigroup by adding all commutative laws to the defining relations of the semigroup. In this case we obtain a commutative semigroup $\mathcal{G}_{0}$ of order eight: it has three idempotent generators $c, l, m$ satisfying the relations $c l=l c, c m=m c$ and $l m=m l$, and consists of the elements $\mathbf{1}, c, l, m, c l, c m, l m, c l m=\mathbf{0}$. 
It is easy to represent $\mathcal{G}_{0}$ by matrices: we define

$$
C_{0}=\left(\begin{array}{lll}
1 & 0 & 0 \\
0 & 1 & 0 \\
0 & 0 & 0
\end{array}\right), \quad L_{0}=\left(\begin{array}{ccc}
1 & 0 & 0 \\
0 & 0 & 0 \\
0 & 0 & 1
\end{array}\right), \quad M_{0}=M=\left(\begin{array}{lll}
0 & 0 & 0 \\
0 & 1 & 0 \\
0 & 0 & 1
\end{array}\right) .
$$

These matrices define orthogonal projections of $\mathbf{R}^{3}$ onto the two-dimensional coordinate planes $x_{3}=0, x_{2}=0$, and $x_{1}=0$, respectively; $C$ and $L$ map $\mathbf{R}^{3}$ obliquely onto the same planes. Obviously $C$ and $L$ are perturbations of $C_{0}$ and $L_{0}$, and the semigroup $\mathcal{G}$ can be viewed as a perturbation of its abelization $\mathcal{G}_{0}$.

It is not possible to use $2 \times 2$ matrices in the representation in Theorem 3.3. This is because for $2 \times 2$ real or complex matrices $A$ and $B$ such that $A$ and $B A$ are idempotent, either $A B$ is idempotent or $B A=0$. Therefore, with $2 \times 2$ matrices $C, L$, and $M$ such that $C, M C$, and $L C$ are idempotent whereas $C M$ and $C L$ are not, we must have $M C=L C=0$ : the representation cannot be faithful.

\section{The Semigroup Generated By the three Closure operators}

As stated in Proposition 2.5, the three operators $c, l, m$ defined on the set $[-\infty,+\infty]^{E}$ of all functions on a topological vector space $E$ are idempotent, i.e., they satisfy (2.7). They are closure operators, since the other requirements for being a closure operator are obviously satisfied. Moreover, they satisfy the relations (2.8) and (2.9). The semigroup $G(E)$ they generate consists of all compositions $f_{k} \circ \cdots \circ f_{2} \circ f_{1}$, where $k \geqslant 0$ and $f_{j}=c, l, m$ for $j=1, \ldots, k$. This semigroup is a homomorphic image of the abstract semigroup $\mathcal{G}$ constructed in Section 3 . We shall now determine its structure for all finite-dimensional topological vector spaces as well as for all normed spaces.

If $E$ is a topological vector space of finite dimension, then $\overline{\{0\}}$, the closure of the origin, is a vector subspace and the quotient $E / \overline{\{0\}}$ is separated (Hausdorff). The space is isomorphic to $\overline{\{0\}} \times(E / \overline{\{0\}}) \cong\left(\mathbf{R}^{k}\right)_{\chi} \times \mathbf{R}^{n-k}$, where the index $\chi$ indicates that we equip $\mathbf{R}^{k}$ with the chaotic topology, i.e., the topology such that the only neighborhood of the origin is the whole space. The dimensions of $E$ and $\overline{\{0\}}$ determine the semigroup $G(E)$; equivalently, the dimension of the algebraic dual $E^{*}$ and that of the topological dual $E^{\prime}$ determine the semigroup, since $\operatorname{dim} E^{\prime}$ is equal to the codimension of $\overline{\{0\}}$ in the finite-dimensional case.

Theorem 4.1. On any real topological vector space $E$ the three closure operators $c, l, m$ defined by (1.1)-(1.3) generate a semigroup $G(E)$ of at most eighteen elements. They can be represented as compositions of $c, l, m$ as follows (for simplicity we write composition as juxtaposition):

(0) One element with zero factors: id $=\mathbf{1}$;

(i) Three elements with one factor: $c, l, m$;

(ii) Six element with two factors: $c l, c m, l c, l m, m c, m l$;

(iii) Six elements with three factors: $\mathrm{clm}, \mathrm{cml}, \mathrm{lcm}, \mathrm{lmc}, \mathrm{mcl}, \mathrm{mlc}=\mathbf{0}$;

(iv) Two elements with four factors: lcml, lmcl.

Of these, the elements represented by $\mathrm{id}=\mathbf{1}, c, l, m, l c, m c, m l, m l c=\mathbf{0}$ (not necessarily distinct) are closure operators.

$\left(\mathrm{A}_{1}\right)$ If $E$ has dimension zero, then $G(E)$ consists of only one element. 
$\left(\mathrm{A}_{15}\right)$ If $E=\mathbf{R}$ with the usual topology, then $G(E)$ has fifteen elements; we have $l m c=l m c l=m c l=\mathbf{0}$ and all other elements are different from $\mathbf{0}$ and each other.

$\left(\mathrm{A}_{16}\right)$ If $E=\mathbf{R}^{n}, n \geqslant 2$, with the usual topology, then $G(E)$ has sixteen elements; we have $l m c=l m c l=\mathbf{0}$ and all other elements are different from $\mathbf{0}$ and each other.

$\left(\mathrm{B}_{6}\right)$ If $E$ is nonzero and equipped with the chaotic topology, then $G(E)$ has six elements, 1, c, $m, \mathrm{~cm}, \mathrm{mc}$, and $\mathrm{l}$, the last being equal to all other products.

$\left(\mathrm{B}_{16}\right)$ If $E$ is of finite dimension and $\overline{\{0\}}$ has codimension one and dimension at least one, then $G(E)$ has sixteen elements; we have $m c l=l m c l=\mathbf{0}$ and all other elements are different from $\mathbf{0}$ and each other.

$\left(\mathrm{B}_{17}\right)$ If $E$ is finite-dimensional and $\overline{\{0\}}$ has codimension at least two and dimension at least one, then $G(E)$ has seventeen elements; we have lmcl $=\mathbf{0}$ and all other elements are different from $\mathbf{0}$ and each other.

(C) Finally, if $E$ is a normed space of infinite dimension, then $G(E)$ has eighteen elements and so is isomorphic to $\mathcal{G}$ as defined in Definition 3.1.

To sum up, the order of $G(E)$ in the finite-dimensional case is $1,6,15,16$ or 17 according to the following table, where $n=\operatorname{dim} E$ and $k=\operatorname{dim} \overline{\{0\}}$.

$\begin{array}{rrrrrrrr} & k & 0 & 1 & 2 & 3 & 4 & 5 \\ n & & & & & & & \\ 0 & & 1 & & & & & \\ 1 & & 15 & 6 & & & & \\ 2 & & 16 & 16 & 6 & & & \\ 3 & & 16 & 17 & 16 & 6 & & \\ 4 & & 16 & 17 & 17 & 16 & 6 & \\ 5 & & 16 & 17 & 17 & 17 & 16 & 6\end{array}$

Proof. The proof is organized as follows. We first present examples to show that the number of elements in $G(E)$ is a least as large as that indicated in the statement of the theorem. Then we shall prove that in finite-dimensional spaces, the number is not larger than stated.

Example 4.2. Define a function $f_{1}$ on the real line by putting $f_{1}(x)=+\infty$ for $x<0, f_{1}(0)=1$, and $f_{1}(x)=-x^{2}$ for $x>0$. Then $G(\mathbf{R})\left(f_{1}\right)$ consists of the following six different functions:

$$
\begin{gathered}
f_{1}=m\left(f_{1}\right), \quad c\left(f_{1}\right)=c m\left(f_{1}\right), \quad l\left(f_{1}\right)=\operatorname{lm}\left(f_{1}\right)=m l\left(f_{1}\right), \\
c l\left(f_{1}\right)=\operatorname{clm}\left(f_{1}\right)=\operatorname{cml}\left(f_{1}\right), \quad l c\left(f_{1}\right)=\operatorname{lcm}\left(f_{1}\right)=\operatorname{lcml}\left(f_{1}\right), \\
m c\left(f_{1}\right)=\operatorname{lmc}\left(f_{1}\right)=\operatorname{lmcl}\left(f_{1}\right)=\operatorname{mcl}\left(f_{1}\right)=m l c\left(f_{1}\right)=-\infty .
\end{gathered}
$$

Example 4.3. Let $E=\mathbf{R}$ with the usual topology and define $f_{2}(x)=+\infty$ for $x<0, f_{2}(0)=0$, and $f_{2}(x)=\log x$ for $x>0$. Then $f_{2}$ does not assume the value $-\infty$, so $m\left(f_{2}\right)=f_{2}, c m\left(f_{2}\right)=c\left(f_{2}\right)$. We see that $G(\mathbf{R})\left(f_{2}\right)$ consists of five different functions, viz.

$$
\begin{gathered}
f_{2}=m\left(f_{2}\right), \quad c\left(f_{2}\right)=c m\left(f_{2}\right), \quad l\left(f_{2}\right)=\operatorname{lm}\left(f_{2}\right), \\
c l\left(f_{2}\right)=c l m\left(f_{2}\right)=l c\left(f_{2}\right)=l c m\left(f_{2}\right), \\
m l\left(f_{2}\right)=m c\left(f_{2}\right)=\operatorname{cml}\left(f_{2}\right)=\operatorname{lmc}\left(f_{2}\right)=m c l\left(f_{2}\right) \\
=l c m l\left(f_{2}\right)=\operatorname{lmcl}\left(f_{2}\right)=m l c\left(f_{2}\right),
\end{gathered}
$$


where the last function is equal to minus infinity identically.

Example 4.4. Define $f_{3}$ on the real line by putting $f_{3}(x)=+\infty$ for $x<0$; $f_{3}(0)=1$, and $f_{3}(x)=0, x>0$. Then $G(\mathbf{R})\left(f_{3}\right)$ consists of two functions, viz.

$$
f_{3}=c\left(f_{3}\right)=m\left(f_{3}\right)=c m\left(f_{3}\right)=m c\left(f_{3}\right),
$$

and the function $l\left(f_{3}\right)$, which is also the result of applying all other operators to $f_{3}$.

Example 4.5. Define $f_{4}$ on $\mathbf{R}$ by putting $f_{4}(x)=+\infty$ for negative $x, f_{4}(0)=-\infty$, and $f_{4}(x)=0$ for $x$ positive. Then $G(\mathbf{R})$ consists of three functions, viz.

$$
f_{4}=l\left(f_{4}\right), \quad c\left(f_{4}\right)=\operatorname{cl}\left(f_{4}\right)=l c\left(f_{4}\right),
$$

and the function $-\infty$, which is the result of applying all other elements to $f_{4}$.

Combining Examples 4.2-4.5, we see that there are at least fifteen elements in $G(\mathbf{R})$, the only elements which have not been isolated being the four elements $l m c$, $m c l, l m c l$ and $m l c=\mathbf{0}$. Indeed, these four elements always give the same result when $E=\mathbf{R}$, as we shall prove in Proposition 4.11. Let us now look at examples in two variables.

Example 4.6. Let $E=\mathbf{R}^{2}$ with the usual topology and define $g_{1}$ by the requirement that $g_{1}(x)=0$ when $x_{2}>0$ and $\left|x_{1}\right| x_{2}=1$, and with the value $+\infty$ otherwise. Then $g_{1}$ is lower semicontinuous, so $l\left(g_{1}\right)=g_{1}$, but $c\left(g_{1}\right)(x)$ is zero when $x_{2}>0$ and $+\infty$ otherwise, thus $l c\left(g_{1}\right) \neq c\left(g_{1}\right)$. We see that $G\left(\mathbf{R}^{2}\right)\left(g_{1}\right)$ consists of three different functions, viz.

$$
\begin{gathered}
g_{1}=l\left(g_{1}\right)=m\left(g_{1}\right)=\operatorname{lm}\left(g_{1}\right)=m l\left(g_{1}\right), \\
c\left(g_{1}\right)=c l\left(g_{1}\right)=\operatorname{cm}\left(g_{1}\right)=\operatorname{clm}\left(g_{1}\right)=m c\left(g_{1}\right)=\operatorname{cml}\left(g_{1}\right)=\operatorname{mcl}\left(g_{1}\right), \\
l c\left(g_{1}\right)=\operatorname{lcm}\left(g_{1}\right)=\operatorname{lmc}\left(g_{1}\right)=\operatorname{lcml}\left(g_{1}\right)=\operatorname{lmcl}\left(g_{1}\right)=\operatorname{mlc}\left(g_{1}\right) .
\end{gathered}
$$

In particular, $l m c l$ and $m c l$ yield different results. Together with Examples 4.2-4.5, this shows that there are at least sixteen elements in $G\left(\mathbf{R}^{2}\right)$.

Example 4.7. Let $E=\mathbf{R}^{2}$ be equipped with the topology generated by the seminorm $x \mapsto\left|x_{2}\right|$. Define a function on $E$ by putting $g_{2}(x)=+\infty$ when $x_{2} \leqslant 0$, and $g_{2}(x)=x_{1}$ when $x_{2}>0$. Then $g_{2}$ is not lower semicontinuous, and $l\left(g_{2}\right)$ takes the value $-\infty$ without being $-\infty$ identically. We see that $G(E)\left(g_{2}\right)$ consists of three different functions, viz.

$$
\begin{gathered}
g_{2}=c\left(g_{2}\right)=m\left(g_{2}\right)=c m\left(g_{2}\right)=m c\left(g_{2}\right), \\
l\left(g_{2}\right)=c l\left(g_{2}\right)=\operatorname{lm}\left(g_{2}\right)=\operatorname{clm}\left(g_{2}\right)=\operatorname{lc}\left(g_{2}\right)=\operatorname{lcm}\left(g_{2}\right)=\operatorname{lmc}\left(g_{2}\right), \\
m l\left(g_{2}\right)=\operatorname{cml}\left(g_{2}\right)=\operatorname{lcml}\left(g_{2}\right)=\operatorname{mcl}\left(g_{2}\right)=\operatorname{lmcl}\left(g_{2}\right)=\operatorname{mlc}\left(g_{2}\right)=-\infty .
\end{gathered}
$$

The function $g_{2}$ shows that $l m c$ is not equal to $l m c l$ nor to $m l c$ in this case.

Let us finally look at an infinite-dimensional space.

Example 4.8. Let $E$ be a normed space of infinite dimension and fix a closed hyperplane $H$ in $E$. There exists a strictly increasing sequence $\left(E_{j}\right)_{0}^{\infty}$ of subspaces of $H$. Pick points $p_{j}, q_{j} \in E_{j} \backslash E_{j-1}$ of norms $\left\|p_{j}\right\|=1 / j$ and $\left\|q_{j}\right\|=j, j \geqslant 2$. We define a function $g_{3}$ as being equal to $-k$ at the points $p_{2 k} \pm q_{2 k+1}$ and equal to $+\infty$ everywhere else, $k \geqslant 1$. Since the set of all points $p_{2 k} \pm q_{2 k+1}$ is closed, this function is lower semicontinuous: $l\left(g_{3}\right)=g_{3}$. We note that the midpoint 
$p_{2 k}=\frac{1}{2}\left(p_{2 k}+q_{2 k+1}\right)+\frac{1}{2}\left(p_{2 k}-q_{2 k+1}\right)$ tends to the origin as $k \rightarrow+\infty$, and that $c\left(g_{3}\right)\left(p_{2 k}\right) \leqslant-k \rightarrow-\infty$; hence $l c\left(g_{3}\right)(0)=-\infty$. On the other hand, the origin does not belong to the convex hull of the points $p_{2 k} \pm q_{2 k+1}$, so $c\left(g_{3}\right)(0)=+\infty$, which shows that $c\left(g_{3}\right)$ cannot be lower semicontinuous: we must have $l c\left(g_{3}\right) \neq c\left(g_{3}\right)$. Moreover, $l c\left(g_{3}\right)(x)=+\infty$ when $x \notin H$, so that $l c\left(g_{3}\right) \neq m l c\left(g_{3}\right)$.

To sum up, $G(E)\left(g_{3}\right)$ consists of four different functions:

$$
\begin{gathered}
g_{3}=l\left(g_{3}\right)=m\left(g_{3}\right)=\operatorname{lm}\left(g_{3}\right)=m l\left(g_{3}\right), \\
c\left(g_{3}\right)=c l\left(g_{3}\right)=c m\left(g_{3}\right)=c l m\left(g_{3}\right)=m c\left(g_{3}\right)=c m l\left(g_{3}\right)=m c l\left(g_{3}\right), \\
l c\left(g_{3}\right)=l c m\left(g_{3}\right)=\operatorname{lmc}\left(g_{3}\right)=l c m l\left(g_{3}\right)=\operatorname{lmcl}\left(g_{3}\right), \quad m l c\left(g_{3}\right),
\end{gathered}
$$

where the last is equal to minus infinity identically.

Thus we have finally found a space where the zero element $m l c$ can be separated from all the other elements.

Examples 4.2-4.6 are available to show that all elements separated in $G\left(\mathbf{R}^{2}\right)$ are also separated in the semigroup $G(E)$. To show this we consider $g(x)=f_{j}\left(\xi_{1}(x)\right)$, $j=1, \ldots 4$, and $g(x)=g_{1}\left(\xi_{1}(x), \xi_{2}(x)\right)$, where $\xi_{1}$ and $\xi_{2}$ are two linearly independent continuous linear forms on $E$. However, we need to separate also $l m c$ and $l m c l$ as in Example 4.7. This can be done using $g(x)=g_{2}\left(\eta(x), \xi_{1}(x)\right)$, where $\eta$ is a discontinuous linear form on $E$, thus $\eta \in E^{*} \backslash E^{\prime}$. Such a form always exists, at least if we admit the existence of Hamel bases. Define first $\theta\left(p_{j}\right)=1$, and then extend $\theta$ to the union of all $E_{j}$ by linearity. Then, using a Hamel basis, $\theta$ can be extended to a linear form $\eta$ on all of $E$. Since $\eta\left(p_{j}\right)=\theta\left(p_{j}\right)=1$ and $\left\|p_{j}\right\|=1 / j, \eta$ is discontinuous.

With all these examples in mind we can now finish the proof. Examples $4.2-$ 4.5 show that the number of different elements is at least that stated in the case $\left(\mathrm{A}_{15}\right)$. Combined with Example 4.6 they show the same thing in case $\left(\mathrm{A}_{16}\right)$. With Example 4.8 we can complete the proof of $(\mathrm{C})$, since $G(E)$ can never have more than eighteen elements. We shall now see that there are not more elements in the other cases.

Proposition 4.9. Let $E$ be a topological vector space of finite dimension. Then $\operatorname{lmcl}(f)=\operatorname{mlc}(f)$ for all functions $f: E \rightarrow[-\infty,+\infty]$. If the topology is separated, then $\operatorname{lmc}(f)=\operatorname{lmcl}(f)=\operatorname{mlc}(f)$ for all functions. More generally, the last conclusion holds whenever $E$ is a topological space such that all linear forms are continuous.

Lemma 4.10. If $E$ is a finite-dimensional topological vector space and if $c l(f)>$ $-\infty$, then $l c(f)>-\infty$. If we assume the topology to be separated, then $c(f)>-\infty$ implies that also $l c(f)>-\infty$. The last conclusion holds whenever $E$ is a topological vector space such that all linear forms are continuous.

Proof. It is classical that a convex function on a vector space which does not take the value $-\infty$ admits an affine minorant. In case $E^{*}=E^{\prime}$, this affine function is continuous, so it is also a minorant of $l c(f)$.

In the general case we know that $c l(f)>-\infty$, so, again, there is an affine function $\varphi$ such that $c l(f) \geqslant \varphi$, hence $l c(f)=l c l(f) \geqslant l(\varphi)$. The affine function is given by $\varphi(x)=\varphi(0)+\xi(x)$, where $\xi$ is a linear form on $E$.

If $c l(f)$ is identically $+\infty$, then also $l c(f)$ is identically $+\infty$, and so does not take the value $-\infty$. 
If, on the other hand, there exists a point a such that $\operatorname{cl}(f)(a)<+\infty$, then we use the fact that $c l(f)$ is constant along the cosets of $\overline{\{0\}}$, meaning that $+\infty>$ $c l(f)(a)=c l(f)(a+x) \geqslant \varphi(a+x)=\varphi(0)+\xi(a+x)$ for all $x \in \overline{\{0\}}$. Hence $\xi$ is constant on the cosets of $\overline{\{0\}}$, implying that it is continuous for the given topology on $E$. Thus $c l(f) \geqslant \varphi$ implies that $l c(f)=l c l(f) \geqslant l(\varphi)=\varphi$, so that $l c(f)$ cannot take the value $-\infty$. This proves the lemma.

Proof of Proposition 4.9. Assume first that $c l(f)$ takes the value $-\infty$. Then $m c l(f)$ is identically $-\infty$, and since $\operatorname{mcl}(f) \geqslant \operatorname{lmcl}(f) \geqslant \operatorname{mlc}(f)$, all three functions are equal to minus infinity. Next, if $c l(f)$ does not take the value $-\infty$, then neither does $l c(f)$ (Lemma 4.10), so $m c l(f)=c l(f)$ and $m l c(f)=l c(f)$, and we obtain $\operatorname{lmcl}(f)=l c l(f)=l c(f)=m l c(f)$, as required.

In case $E^{*}=E^{\prime}$, we assume first that $c(f)$ takes the value $-\infty$. Then $m c(f)$ is identically minus infinity, as well as $\operatorname{lmc}(f), \operatorname{lmcl}(f)$, and $m l c(f)$, so the three functions mentioned in the statement of the proposition are all equal. On the other hand, if $c(f)>-\infty$, then by Lemma $4.10, l c(f)>-\infty$, so that $m l c(f)=l c(f)$. We always have $m l c(f) \leqslant \operatorname{lmcl}(f) \leqslant l m c(f) \leqslant l c(f)$, so if $m l c(f)=l c(f)$, then all elements in this chain are equal. This proves Proposition 4.9.

Proposition 4.11. If $E=\mathbf{R}$ with the usual topology, then $\operatorname{lmc}(f)=\operatorname{mcl}(f)=$ $\operatorname{lmcl}(f)=m l c(f)$ for all functions $f: \mathbf{R} \rightarrow[-\infty,+\infty]$.

Lemma 4.12. If $E=\mathbf{R}$ with the usual topology, then $c(f)>-\infty$ implies that $l c(f)=c l(f)$.

Proof. If $f$ is any function of one real variable such that $c(f)>-\infty$, then $c(f)$ is finite, hence continuous, in an open interval $I$, and equal to plus infinity in the complement of its closure. We therefore have $l c(f)=c(f)$ in $I \cup \complement \bar{I}$, as well as $c(f) \geqslant c l(f) \geqslant l c(f)$ everywhere, which implies that $l c(f)=c l(f)$ in $I \cup \complement \bar{I}$. The endpoints of $I$ remain to be studied. For definiteness, let $I=] 0, b[$ and assume that $l c(f)(0)<c l(f)(0)$, say $l c(f)(0)=0<2 \varepsilon=c l(f)(0) \leqslant l(f)(0)$. Then there exists a positive number $\delta$ such that $f(x) \geqslant \varepsilon$ for all $x$ with $|x| \leqslant \delta$. We may of course take $\delta<b$. Define an auxiliary function $g$ to be equal to $+\infty$ for $x<0$, to take the value $\varepsilon$ at the origin and the value $\min (c(f)(\delta), \varepsilon)$ at the point $\delta$, to be affine in the interval $[0, \delta]$, and finally to be equal to $(x / \delta) \min (c(f)(\delta), \varepsilon)$ for $x>\delta$. The function $g$ is convex and a minorant of $f$, so we get $g=c(g) \leqslant c(f)$. Then $\varepsilon=l c(g)(0) \leqslant l c(f)(0)$, contradicting the assumption that $l c(f)=0$.

Proof of Proposition 4.11. Comparing with Proposition 4.9, we see that it only remains to be proved that $\operatorname{mcl}(f)$ is equal to the three other functions. If $c l(f)$ assumes the value $-\infty$, then $\operatorname{mcl}(f)$ is $-\infty$ identically, so we are done. If, on the other hand, $c l(f)>-\infty$, then by Lemma $4.12, l c(f)=c l(f)$, so $m c l(f)=m l c(f)$ also in this case.

Corollary 4.13. If $E$ is a topological vector space such that $\overline{\{0\}}$ has codimension one, then $m c l=l m c l=\mathbf{0}$.

Proof. For any function $f$ on $E$ we define $g=l(f)$ and note that it is constant on the cosets of $\overline{\{0\}}$, hence a function of one variable. We apply Proposition 4.11 to it and obtain $\operatorname{lmc}(g)=\operatorname{mcl}(g)=\operatorname{lmcl}(g)=m l c(g)$. Inserting the definition of $g$, we get $\operatorname{lmcl}(f)=\operatorname{mcl}(f)=\operatorname{mlc}(f)$, as claimed. 
We can now finish the proof of Theorem 4.1. If $E=\{0\}$, all elements in $G(E)$ are equal to the identity, so $\left(\mathrm{A}_{1}\right)$ follows. On the basis of Examples 4.2-4.5 and Proposition 4.11 we can finish the proof in case $\left(\mathrm{A}_{15}\right)$. Similarly, case $\left(\mathrm{A}_{16}\right)$ follows from Examples 4.2-4.6 and Proposition 4.9.

If $E$ is equipped with the chaotic topology, then $b l a(f)$ is a constant function for all elements $a, b \in G(E)$; moreover, it is the same constant. Therefore $G(E)(f)$ cannot contain more than six different functions, viz. $f, c(f), m(f), c m(f), m c(f)$, and $l(f)$. On the other hand, if $E$ is nonzero, then Examples 4.3-4.5, where we now use the chaotic topology, show that $\mathbf{1}, c, m, c m, m c$, and $l$ are actually different. So $\left(\mathrm{B}_{6}\right)$ is proved.

Examples 4.2-4.5 and the function $g_{2}$ constructed in Example 4.7 show that there are at least sixteen elements in case $\left(\mathrm{B}_{16}\right)$ - however, not the same sixteen elements as in case $\left(\mathrm{A}_{16}\right)$. Corollary 4.13 then shows that the number cannot exceed sixteen. If the codimension of $\overline{\{0\}}$ is at least two, we may use the function $g_{2}$ constructed in Example 4.7 together with all the functions in Examples 4.2-4.6 to show that the number is at least seventeen; Proposition 4.9 then shows that there are not more that seventeen elements in case $\left(\mathrm{B}_{17}\right)$. Finally, as already remarked, case $(\mathrm{C})$ is dealt with by Examples 4.2-4.5, 4.7, and 4.8, since they show that there are at least eighteen elements in $G(E)$ in that case.

\section{The Multiplicative StruCture of THE SEMigroup}

The multiplicative structure of the semigroup $\mathcal{G}$ is as shown in Table 1.

The semigroup is highly noncommutative. To turn that statement into something quantitative, we may argue as follows. We must have $a b=b a$ if $a$ and $b$ are equal, or if one of them is equal to $\mathbf{0}$ or $\mathbf{1}$. There are 84 such pairs; they commute automatically. The remaining pairs $a, b$ with $b$ following $a$ in the list are 120 in number; of those there are 90 pairs such that $a b \neq b a$. In other words, out of the

TABLE 1. The multiplication table of the semigroup.

$\begin{array}{cccccccccccccccccc}\mathbf{1} & c & l & m & c l & c m & l m & c l m & l c & m c & m l & l c m & c m l & l c m l & l m c & m c l & l m c l & \mathbf{0} \\ c & c & c l & c m & c l & c m & c l m & c l m & l c & m c & c m l & l c m & c m l & l c m l & l m c & m c l & l m c l & \mathbf{0} \\ l & l c & l & l m & l c & l c m & l m & l c m & l c & l m c & m l & l c m & l c m l & l c m l & l m c & l m c l & l m c l & \mathbf{0} \\ m & m c & m l & m & m c l & m c & m l & m c l & \mathbf{0} & m c & m l & \mathbf{0} & m c l & \mathbf{0} & \mathbf{0} & m c l & \mathbf{0} & \mathbf{0} \\ c l & l c & c l & c l m & l c & l c m & c l m & l c m & l c & l m c & c m l & l c m & l c m l & l c m l & l m c & l m c l & l m c l & \mathbf{0} \\ c m & m c & c m l & c m & m c l & m c & c m l & m c l & \mathbf{0} & m c & c m l & \mathbf{0} & m c l & \mathbf{0} & \mathbf{0} & m c l & \mathbf{0} & \mathbf{0} \\ l m & l m c & m l & l m & l m c l & l m c & m l & l m c l & \mathbf{0} & l m c & m l & \mathbf{0} & l m c l & \mathbf{0} & \mathbf{0} & l m c l & \mathbf{0} & \mathbf{0} \\ c l m & l m c & c m l & c l m & l m c l & l m c & c m l & l m c l & \mathbf{0} & l m c & c m l & \mathbf{0} & l m c l & \mathbf{0} & \mathbf{0} & l m c l & \mathbf{0} & \mathbf{0} \\ l c & l c & l c & l c m & l c & l c m & l c m & l c m & l c & l m c & l c m l & l c m & l c m l & l c m l & l m c & l m c l & l m c l & \mathbf{0} \\ m c & m c & m c l & m c & m c l & m c & m c l & m c l & \mathbf{0} & m c & m c l & \mathbf{0} & m c l & \mathbf{0} & \mathbf{0} & m c l & \mathbf{0} & \mathbf{0} \\ m l & \mathbf{0} & m l & m l & \mathbf{0} & \mathbf{0} & m l & \mathbf{0} & \mathbf{0} & \mathbf{0} & m l & \mathbf{0} & \mathbf{0} & \mathbf{0} & \mathbf{0} & \mathbf{0} & \mathbf{0} & \mathbf{0} \\ l c m & l m c & l c m l & l c m & l m c l & l m c & l c m l & l m c l & \mathbf{0} & l m c & l c m l & \mathbf{0} & l m c l & \mathbf{0} & \mathbf{0} & l m c l & \mathbf{0} & \mathbf{0} \\ c m l & \mathbf{0} & c m l & c m l & \mathbf{0} & \mathbf{0} & c m l & \mathbf{0} & \mathbf{0} & \mathbf{0} & c m l & \mathbf{0} & \mathbf{0} & \mathbf{0} & \mathbf{0} & \mathbf{0} & \mathbf{0} & \mathbf{0} \\ l c m l & \mathbf{0} & l c m l & l c m l & \mathbf{0} & \mathbf{0} & l c m l & \mathbf{0} & \mathbf{0} & \mathbf{0} & l c m l & \mathbf{0} & \mathbf{0} & \mathbf{0} & \mathbf{0} & \mathbf{0} & \mathbf{0} & \mathbf{0} \\ l m c & l m c & l m c l & l m c & l m c l & l m c & l m c l & l m c l & \mathbf{0} & l m c & l m c l & \mathbf{0} & l m c l & \mathbf{0} & \mathbf{0} & l m c l & \mathbf{0} & \mathbf{0} \\ m c l & \mathbf{0} & m c l & m c l & \mathbf{0} & \mathbf{0} & m c l & \mathbf{0} & \mathbf{0} & \mathbf{0} & m c l & \mathbf{0} & \mathbf{0} & \mathbf{0} & \mathbf{0} & \mathbf{0} & \mathbf{0} & \mathbf{0} \\ l m c l & \mathbf{0} & l m c l & l m c l & \mathbf{0} & \mathbf{0} & l m c l & \mathbf{0} & \mathbf{0} & \mathbf{0} & l m c l & \mathbf{0} & \mathbf{0} & \mathbf{0} & \mathbf{0} & \mathbf{0} & \mathbf{0} & \mathbf{0} \\ \mathbf{0} & \mathbf{0} & \mathbf{0} & \mathbf{0} & \mathbf{0} & \mathbf{0} & \mathbf{0} & \mathbf{0} & \mathbf{0} & \mathbf{0} & \mathbf{0} & \mathbf{0} & \mathbf{0} & \mathbf{0} & \mathbf{0} & \mathbf{0} & \mathbf{0} & \mathbf{0}\end{array}$


TABLE 2. Listing $a b$ and $b a$ for comparison.

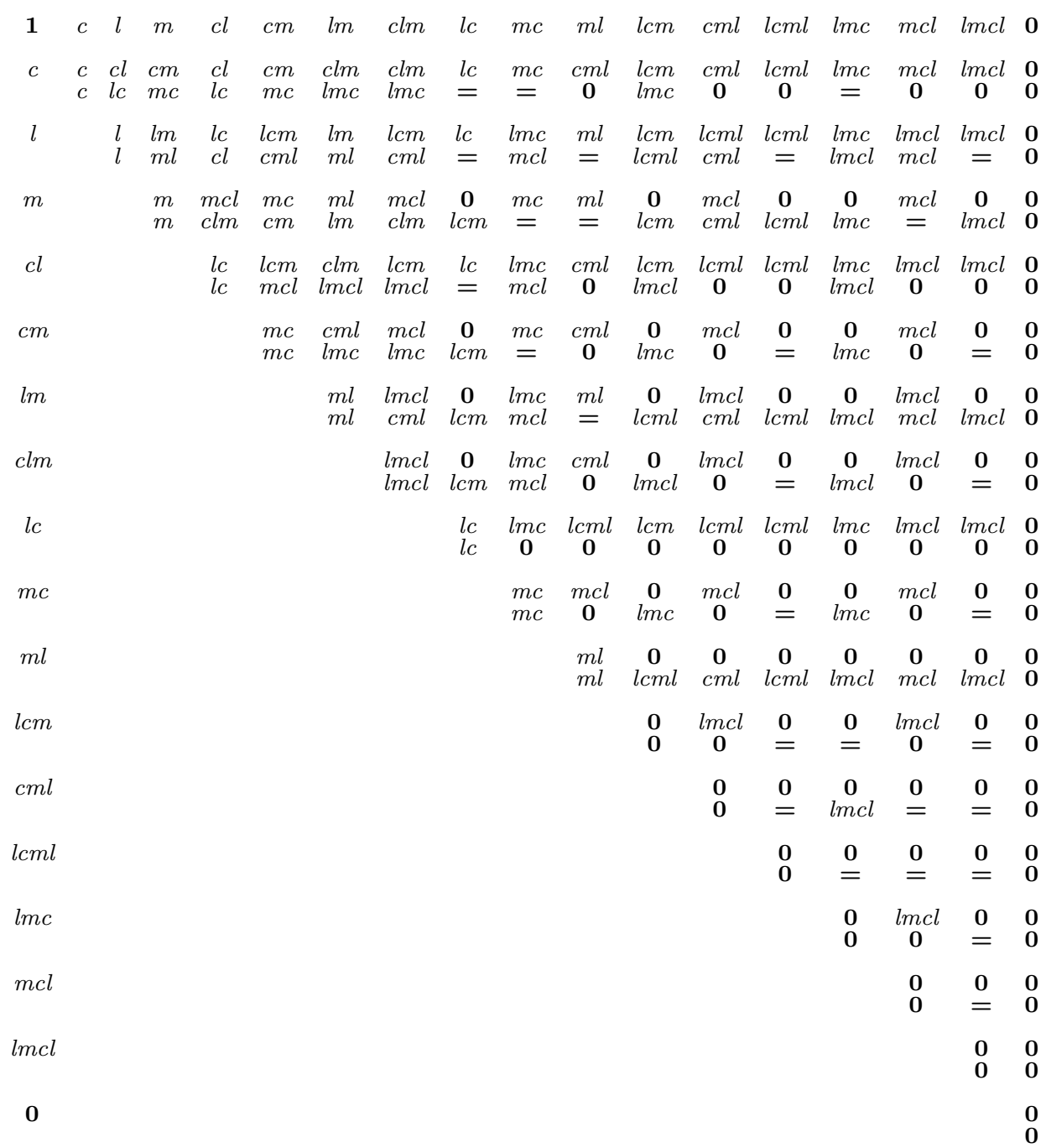

pairs which do not commute automatically, 75 per cent do not commute. In Table 2 the 30 commuting pairs which do not commute automatically are marked by an equality sign.

Another measure of noncommutativity is the order of the abelization $\mathcal{G}_{0}$ (eight) compared with that of $\mathcal{G}$ (eighteen).

\section{The order Structure of The SEMigroup}

For every vector space $E$ we may define an order in the semigroup $G(E)$ by declaring that $a \leqslant b$ for two elements $a, b$ of $G(E)$ if $a(f)(x) \geqslant b(f)(x)$ for all functions $f: E \rightarrow[-\infty,+\infty]$ and all points $x \in E$. (The order between functions is that of inclusion between their epigraphs.) Since $G(E)$ is isomorphic to $\mathcal{G}$ for large spaces $E$, we also get an order in $\mathcal{G}$. 


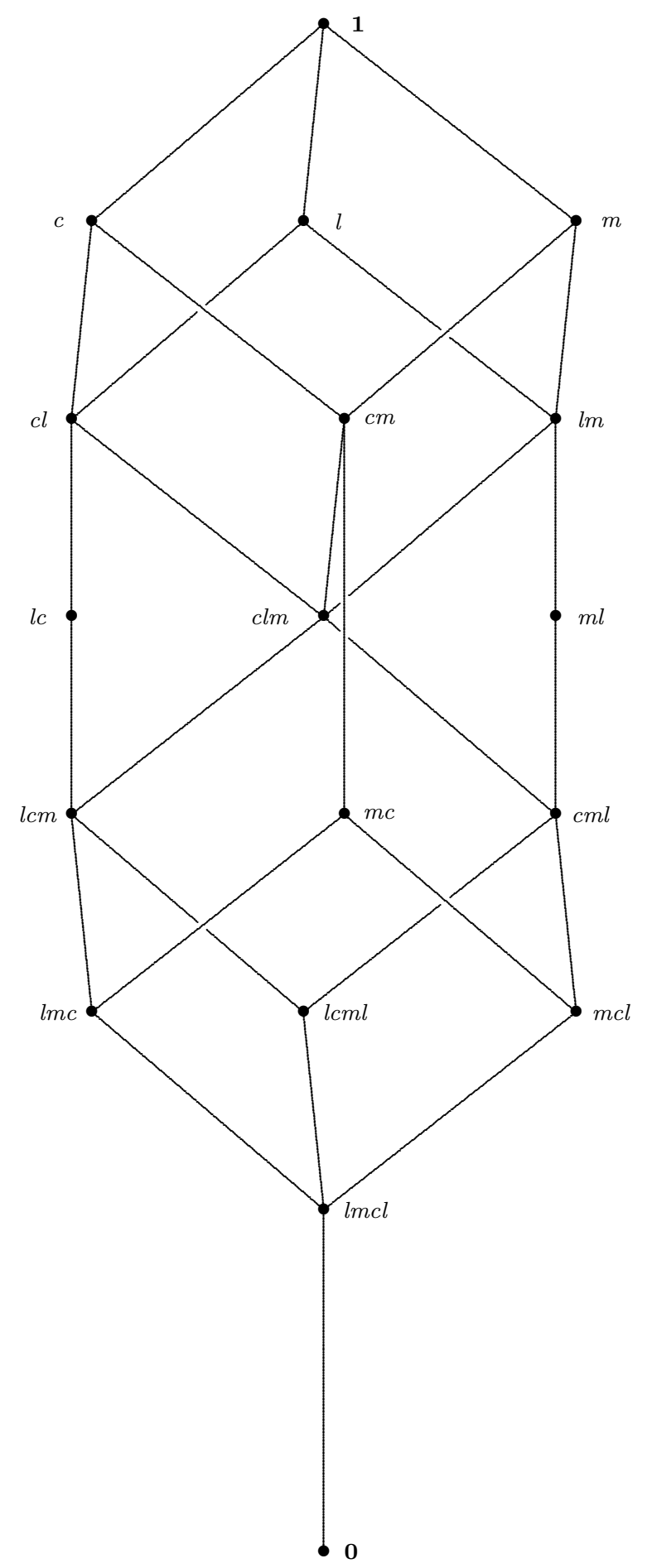

FiguRE 1. The Hasse diagram of the semigroup. 
Thus we see that $\mathbf{1} \leqslant c, l, m \leqslant \mathbf{0}$, and that $c l \leqslant l c, c m \leqslant m c$, and $l m \leqslant m l$. Multiplication preserves order in the sense that $a \leqslant a^{\prime}, b \leqslant b^{\prime}$ implies $a b \leqslant a^{\prime} b^{\prime}$.

The order structure of $\mathcal{G}$ can be read off from its Hasse diagram, which is shown in Figure 1. Of course the geometric aspect of the arrangement is not uniquely determined by the order. In the presentation we have chosen, the semigroup consists basically of two cubes which share a common vertex. The upper cube hangs from the vertex 1, and its bottom vertex $\mathrm{clm}$ is the top vertex of the lower cube, which, however, is not complete in that there is no edge from $\mathrm{clm}$ to $\mathrm{mc}$. In addition to the vertices of the two cubes, there is one elements between $c l$ and $l c m$, viz. $l c$, and one between $l m$ and $\mathrm{cml}$, viz. $\mathrm{ml}$. Finally, the zero element $m l c=\mathbf{0}$ is attached below the bottom vertex $l m c l$ of the lower cube.

The upper cube realizes the abelization $\mathcal{G}_{0}$ of $\mathcal{G}$ mentioned at the end of Section 3.

The whole semigroup possesses a 2-symmetry: it is equal to its mirror image in the plane determined by $\mathbf{1}, \mathbf{0}$ and $l$. Mathematically the symmetry is achieved by interchanging $c$ and $m$ and then reversing the order of all products (the conditions (3.1) and (3.2) are invariant under this change). The upper cube in Figure 1 possesses of course more symmetry, but this does not correspond to any symmetry of the semigroup, only to a symmetry of its abelization $\mathcal{G}_{0}$.

We emphasize that there is no order relation (no edge) between $\mathrm{clm}$ and $\mathrm{mc}$. This follows from Example 4.2, where the function $f_{1}$ shows that we do not have $c l m \geqslant m c$, and Example 4.4, where the function $f_{3}$ shows that we do not have $c l m \leqslant m c$. As a consequence, $G(\mathbf{R})$ is not a lattice. The same is then true of $\mathcal{G}$. In fact, both $l m c$ and $m c l$ are minimal majorants of the set $\{c l m, m c\}$, so there is no smallest majorant of that set. Similarly, the infimum of $l m c$ and $m c l$ does not exist; $m c$ and $c l m$ are both maximal minorants of the set $\{l m c, m c l\}$.

In Table 3 the infimum and the supremum (when they exist) of two elements of the semigroup are listed. Only the upper triangle, corresponding to the case where $a$ precedes $b$ in the listing we have chosen, is entered. When $a \leqslant b$ in the partial order of the semigroup, we have of course $\inf (a, b)=a$ and $\sup (a, b)=b$; in these

cases nothing is written. A star $\star$ indicates that the supremum or infimum does not exist.

\section{Comparison Between the Supremum AND THE PRODUCT OF TWO ELEMENTS}

With the order we have defined in $\mathcal{G}$, it is clear that $a, b \leqslant a b$, thus that $\sup (a, b) \leqslant a b$ when the supremum exists. By symmetry, $\sup (a, b) \leqslant b a$, so that

$$
\sup (a, b) \leqslant \inf (a b, b a)
$$

whenever the supremum and infimum exist. In Table 4 we list, for each pair $a, b$ with $a$ preceding $b, \sup (a, b)$ and $\inf (a b, b a)$ (a nonexisting supremum or infimum is indicated by a star). Let us agree to call a pair regular if we have equality in (7.1). Every pair $(a, b)$ with $a$ or $b$ equal to $\mathbf{0}$ or $\mathbf{1}$ is regular. A pair $(a, a)$ is regular if and only if $a$ is idempotent. There are 136 pairs $(a, b)$ with $a$ preceding $b$ or $a$ equal to $b$ but with none of them equal to $\mathbf{0}$ or $\mathbf{1}$. Of these, there are 90 regular pairs, thus satisfying $\sup (a, b)=\inf (a b, b a)$, marked by an equality sign in Table 4 ; 41 irregular pairs with $\sup (a, b) \neq \inf (a b, b a)$; four pairs where neither $\sup (a, b)$ nor $\inf (a b, b a)$ exists; and one pair where $\sup (a, b)$ exists but $\inf (a b, b a)$ does not exist. 
TABLE 3. The infimum and supremum (when they exist) of two elements of the semigroup.

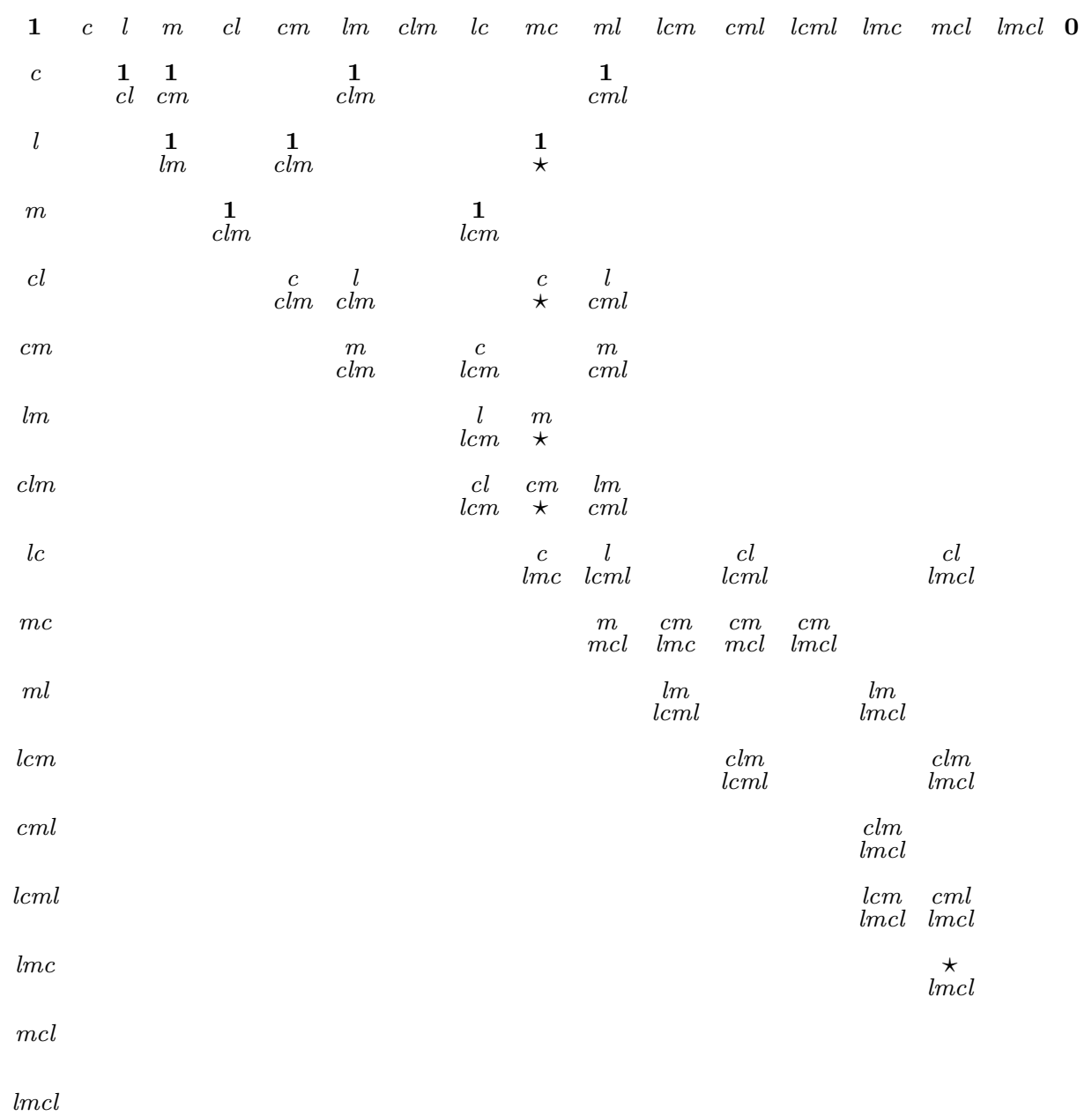


TABle 4. Comparison between $\sup (a, b)$ and $\inf (a b, b a)$.

\begin{tabular}{|c|c|c|c|c|c|c|c|c|c|c|c|c|c|c|c|c|c|}
\hline 1 & $c$ & $l$ & $m$ & $\mathrm{cl}$ & $\mathrm{cm}$ & $l m$ & $\mathrm{clm}$ & $l c$ & $m c$ & $m l$ & $\mathrm{lcm}$ & $\mathrm{cml}$ & $l \mathrm{cml}$ & $\operatorname{lm} c$ & $m c l$ & $l m c l$ & 0 \\
\hline$c$ & $\stackrel{c}{=}$ & $\stackrel{c l}{=}$ & $\stackrel{\mathrm{cm}}{=}$ & $\stackrel{c l}{=}$ & $\stackrel{\mathrm{cm}}{=}$ & $\stackrel{c l m}{=}$ & $\stackrel{c l m}{=}$ & $\stackrel{l c}{=}$ & $\begin{array}{l}m c \\
=\end{array}$ & $\begin{array}{c}\mathrm{cml} \\
=\end{array}$ & $\begin{array}{l}\text { lcm } \\
=\end{array}$ & $\begin{array}{c}c m l \\
=\end{array}$ & $\begin{array}{l}l \mathrm{~cm} l \\
=\end{array}$ & $\stackrel{\operatorname{lm} c}{=}$ & $\begin{array}{c}m c l \\
=\end{array}$ & $\begin{array}{l}\operatorname{lm} c l \\
=\end{array}$ & $\begin{array}{l}\mathbf{0} \\
\mathbf{0}\end{array}$ \\
\hline$l$ & & $\stackrel{l}{=}$ & $\stackrel{l m}{=}$ & $\stackrel{c l}{=}$ & $\stackrel{c l m}{=}$ & $\stackrel{\operatorname{lm}}{=}$ & $\stackrel{c l m}{=}$ & $\stackrel{l c}{=}$ & ᄎ & $\stackrel{m l}{=}$ & $\stackrel{l \mathrm{~cm}}{=}$ & $\stackrel{c m l}{=}$ & $\stackrel{l c m l}{=}$ & $\stackrel{\operatorname{lm} c}{=}$ & $\begin{array}{l}m c l \\
=\end{array}$ & $\stackrel{\operatorname{lm} c l}{=}$ & $\begin{array}{l}\mathbf{0} \\
\mathbf{0}\end{array}$ \\
\hline$m$ & & & $\stackrel{m}{=}$ & $\stackrel{c l m}{=}$ & $\stackrel{\mathrm{cm}}{=}$ & $\stackrel{\operatorname{lm}}{=}$ & $\stackrel{c l m}{=}$ & $\begin{array}{l}l \mathrm{~cm} \\
=\end{array}$ & $\begin{array}{l}m c \\
=\end{array}$ & $\begin{array}{l}m l \\
=\end{array}$ & $\begin{array}{l}\text { lcm } \\
=\end{array}$ & $\stackrel{c m l}{=}$ & $\begin{array}{l}l \mathrm{lcml} \\
=\end{array}$ & $\stackrel{\operatorname{lm} c}{=}$ & $\begin{array}{c}m c l \\
=\end{array}$ & $\begin{array}{l}\operatorname{lm} c l \\
=\end{array}$ & $\begin{array}{l}\mathbf{0} \\
\mathbf{0}\end{array}$ \\
\hline$c l$ & & & & $\begin{array}{l}c l \\
l c\end{array}$ & $\stackrel{c l m}{=}$ & $\stackrel{c l m}{=}$ & $\begin{array}{l}\text { clm } \\
\text { lcm }\end{array}$ & $\stackrel{l c}{=}$ & $\begin{array}{l}\star \\
\star\end{array}$ & $\stackrel{c m l}{=}$ & $\begin{array}{l}l \mathrm{~cm} \\
=\end{array}$ & $\begin{array}{l}\mathrm{cml} \\
\mathrm{lcml}\end{array}$ & $\stackrel{l c m l}{=}$ & $\stackrel{\operatorname{lm} c}{=}$ & $\begin{array}{l}m c l \\
l m c l\end{array}$ & $\begin{array}{l}\operatorname{lm} c l \\
=\end{array}$ & $\begin{array}{l}\mathbf{0} \\
\mathbf{0}\end{array}$ \\
\hline $\mathrm{cm}$ & & & & & $\begin{array}{l}\mathrm{cm} \\
\mathrm{mc}\end{array}$ & $\stackrel{c l m}{=}$ & $\stackrel{c l m}{\star}$ & $\stackrel{l \mathrm{~cm}}{=}$ & $\begin{array}{l}m c \\
=\end{array}$ & $\begin{array}{l}\mathrm{mcl} \\
\mathrm{cml}\end{array}$ & $\begin{array}{l}l \mathrm{~cm} \\
\mathrm{lmc}\end{array}$ & $\begin{array}{l}\mathrm{cml} \\
\mathrm{mcl}\end{array}$ & $\begin{array}{c}l c m l \\
\mathbf{0}\end{array}$ & $\stackrel{\operatorname{lm} c}{=}$ & $\begin{array}{c}m c l \\
=\end{array}$ & $\begin{array}{c}l m c l \\
\mathbf{0}\end{array}$ & $\begin{array}{l}\mathbf{0} \\
\mathbf{0}\end{array}$ \\
\hline$l m$ & & & & & & $\begin{array}{l}l m \\
m l\end{array}$ & $\begin{array}{l}\mathrm{clm} \\
\mathrm{cml}\end{array}$ & $\begin{array}{l}l \mathrm{~cm} \\
=\end{array}$ & ઔ & $\stackrel{m l}{=}$ & $\begin{array}{l}\text { lcm } \\
l \mathrm{cml}\end{array}$ & $\stackrel{c m l}{=}$ & $\stackrel{l c m l}{=}$ & $\begin{array}{l}\operatorname{lm} c \\
\operatorname{lmcl}\end{array}$ & $\begin{array}{c}m c l \\
=\end{array}$ & $\stackrel{\operatorname{lm} c l}{=}$ & $\begin{array}{l}\mathbf{0} \\
\mathbf{0}\end{array}$ \\
\hline $\mathrm{clm}$ & & & & & & & $\begin{array}{c}\mathrm{clm} \\
\mathrm{lmcl}\end{array}$ & $\begin{array}{l}l \mathrm{~cm} \\
=\end{array}$ & ᄎ & $\stackrel{c m l}{=}$ & $\begin{array}{l}\text { lcm } \\
l \mathrm{mcl}\end{array}$ & $\begin{array}{l}\mathrm{cml} \\
\mathrm{lmcl}\end{array}$ & $\begin{array}{c}l c m l \\
\mathbf{0}\end{array}$ & $\begin{array}{l}\operatorname{lmc} c \\
\operatorname{lmcl}\end{array}$ & $\begin{array}{l}m c l \\
l m c l\end{array}$ & $\begin{array}{c}l m c l \\
\mathbf{0}\end{array}$ & $\begin{array}{l}\mathbf{0} \\
\mathbf{0}\end{array}$ \\
\hline$l c$ & & & & & & & & $\stackrel{l c}{=}$ & $\stackrel{\operatorname{lm} c}{=}$ & $\stackrel{l c m l}{=}$ & $\begin{array}{l}l \mathrm{~cm} \\
=\end{array}$ & $\begin{array}{l}l \mathrm{lcml} \\
=\end{array}$ & $\stackrel{l c m l}{=}$ & $\stackrel{\operatorname{lm} c}{=}$ & $\stackrel{\operatorname{lmcl}}{=}$ & $\begin{array}{c}\operatorname{lmcl} \\
=\end{array}$ & $\begin{array}{l}\mathbf{0} \\
\mathbf{0}\end{array}$ \\
\hline$m c$ & & & & & & & & & $\stackrel{m c}{=}$ & $\stackrel{m c l}{=}$ & $\stackrel{\operatorname{lm} c}{=}$ & $\begin{array}{c}m c l \\
=\end{array}$ & $\stackrel{\operatorname{lm} c l}{\mathbf{0}}$ & $\stackrel{\operatorname{lm} c}{=}$ & $\begin{array}{c}m c l \\
=\end{array}$ & $\begin{array}{c}l m c l \\
\mathbf{0}\end{array}$ & $\begin{array}{l}\mathbf{0} \\
\mathbf{0}\end{array}$ \\
\hline$m l$ & & & & & & & & & & $\stackrel{m l}{=}$ & $\begin{array}{l}\text { lcml } \\
=\end{array}$ & $\stackrel{c m l}{=}$ & $\stackrel{l c m l}{=}$ & $\stackrel{\operatorname{lmcl}}{=}$ & $\begin{array}{c}m c l \\
=\end{array}$ & $\begin{array}{c}l m c l \\
=\end{array}$ & $\begin{array}{l}\mathbf{0} \\
\mathbf{0}\end{array}$ \\
\hline lcm & & & & & & & & & & & $\begin{array}{c}l \mathrm{~cm} \\
\mathbf{0}\end{array}$ & $\begin{array}{l}l \mathrm{cml} \\
l m c l\end{array}$ & $\begin{array}{c}l c m l \\
\mathbf{0}\end{array}$ & $\begin{array}{c}\operatorname{lm} c \\
\mathbf{0}\end{array}$ & $\stackrel{\operatorname{lmcl}}{=}$ & $\begin{array}{c}\operatorname{lm} c l \\
\mathbf{0}\end{array}$ & $\begin{array}{l}\mathbf{0} \\
\mathbf{0}\end{array}$ \\
\hline $\mathrm{cml}$ & & & & & & & & & & & & $\stackrel{c m l}{c m}$ & $\begin{array}{c}l c m l \\
\mathbf{0}\end{array}$ & $\stackrel{\operatorname{lmcl}}{=}$ & $\begin{array}{c}m c l \\
\mathbf{0}\end{array}$ & $\begin{array}{c}\operatorname{lm} c l \\
\mathbf{0}\end{array}$ & $\begin{array}{l}\mathbf{0} \\
\mathbf{0}\end{array}$ \\
\hline lcml & & & & & & & & & & & & & $\begin{array}{c}l c m l \\
\mathbf{0}\end{array}$ & $\begin{array}{l}\operatorname{lm} c l \\
\mathbf{0}\end{array}$ & $\begin{array}{c}\operatorname{lm} c l \\
\mathbf{0}\end{array}$ & $\begin{array}{c}l m c l \\
\mathbf{0}\end{array}$ & $\begin{array}{l}\mathbf{0} \\
\mathbf{0}\end{array}$ \\
\hline $\operatorname{lm} c$ & & & & & & & & & & & & & & $\begin{array}{c}\operatorname{lm} c \\
\mathbf{0}\end{array}$ & $\stackrel{\operatorname{lmcl}}{=}$ & $\begin{array}{c}l m c l \\
\mathbf{0}\end{array}$ & $\begin{array}{l}\mathbf{0} \\
\mathbf{0}\end{array}$ \\
\hline$m c l$ & & & & & & & & & & & & & & & $\begin{array}{c}m c l \\
\mathbf{0}\end{array}$ & $\stackrel{\operatorname{lm} c l}{\mathbf{0}}$ & $\begin{array}{l}\mathbf{0} \\
\mathbf{0}\end{array}$ \\
\hline $\operatorname{lmcl}$ & & & & & & & & & & & & & & & & $\stackrel{\operatorname{lm} c l}{\mathbf{0}}$ & $\begin{array}{l}\mathbf{0} \\
\mathbf{0}\end{array}$ \\
\hline 0 & & & & & & & & & & & & & & & & & \\
\hline
\end{tabular}




\section{REFERENCES}

[1948] Birkhoff, Garrett, Lattice Theory. Revised edition. New York: American Mathematical Society. xiii +283 pp., 1948. MR 10:673a

[1944] Everett, C. J., Closure operators and Galois theory in lattices. Trans. Amer. Math. Soc. 55, 1944, 514-525. MR 6:36b

[1955] Hewitt, Edwin, and Zuckerman, Herbert S., Finite dimensional convolution algebras. Acta Math. 93, 1955, 67-119. MR 17:1048h

[1962] Kuroš, A. G., Lekcii po obščej algebre. Moscow: F.M. 396 pp., 1962. MR 25:5097

[1944] Ore, Oystein, Galois connexions. Trans. Amer. Math. Soc. 55, 1944, 493-513. MR 6:36a

Department of Mathematics, Uppsala University, P. O. Box 480, SE-751 06 Uppsala, SWEDEN

E-mail address: kiselman@math.uu.se

URL: http://www.math.uu.se/ ${ }^{\circ i s e l m a n}$ 